\title{
COUGH
}

\section{Development of a symptom specific health status measure for patients with chronic cough: Leicester Cough Questionnaire (LCQ)}

\author{
S S Birring, B Prudon, A J Carr, S J Singh, M D L Morgan, I D Pavord
}

Thorax 2003;58:339-343

See end of article for authors' affiliations

\section{Correspondence to:}

Dr S S Birring, Institute for

Lung Health, Department of

Respiratory Medicine,

Glenfield Hospital,

Leicester LE3 9QP, UK;

sb134@le.ac.uk

Revised version received 6 November 2002

Accepted for publication 27 November 2002

\begin{abstract}
Background: Chronic cough is a common condition which has a significant impact on quality of life.
Assessment and management are hampered by the absence of well validated outcome measures. The
Background: Chronic cough is a common condition which has a significant impact on quality of life.
Assessment and management are hampered by the absence of well validated outcome measures. The development and validation of the Leicester Cough Questionnaire (LCQ), a self-completed health related quality of life measure of chronic cough, is presented.

Methods: Patients with chronic cough were recruited from outpatient clinics. The development of the LCQ consisted of three phases: phase 1 (item generation); phase 2 (item reduction, allocation of items to domains and validation of questionnaire); phase 3 (repeatability and responsiveness testing of final version of questionnaire).

Results: Phase 1: Literature review, multidisciplinary team meeting and 15 structured interviews with chronic cough patients generated 44 items (LCQ1) with a 7 point Likert response scale. Phase 2: 104 chronic cough outpatients completed the LCQ1 along with an importance rating for each item. The clinical impact factor method was used for item reduction to 19 items (LCQ2: final version). These items were divided into three domains (physical, psychological and social) following expert opinion. Internal reliability, as assessed using Cronbach's alpha coefficients, varied between 0.79 and 0.89 . Concurrent validity was high when the LCQ2 $(n=56)$ was compared with a cough visual analogue score $(r=-0.72)$. There was a moderate relationship with response to the St George's Respiratory Questionnaire $(r=-0.54)$ and SF36 total score $(r=0.46)$. Phase 3: Two week repeatability $(n=24)$ was high with intraclass correlation coefficients for domains varying between 0.88 and 0.96 . Responsiveness in nine patients whose cough was successfully treated varied within domains from an effect size of 0.84 to 1.75 .

Conclusion: The $L C Q$ is a valid, repeatable 19 item self-completed quality of life measure of chronic cough which is responsive to change. It should be a useful tool in clinical trials and longitudinal studies.
\end{abstract} ...................

C hronic cough is one of the most common causes of presentation to general practice. At any one time $20 \%$ of the UK population have a troublesome cough and sufferers consume 75 million doses of over the counter antitussive medications annually. ${ }^{1}$ Most cases are acute and self-limiting, although a significant minority are referred for a specialist opinion with an isolated persistent chronic cough. ${ }^{2}$ These patients suffer considerable physical and psychological morbidity. ${ }^{3}$

The assessment of health status is increasingly important in respiratory disease and has been extensively studied in asthma and chronic obstructive pulmonary disease by development of disease specific questionnaires. ${ }^{45}$ Very little is known about the effects of chronic cough on health status because of the lack of such validated questionnaires. Indeed, there is a striking paucity of objective and well validated outcome measures in chronic cough. Our aims were to develop a health related quality of life questionnaire specifically for chronic cough that is brief, simple to administer and score, suitable to monitor individual patients, assess different aspects of health affected in patients, be sensitive enough to detect changes in health status within an adult chronic cough population, and be an outcome measure in clinical trials of new antitussive agents. This study describes the development and validation of the Leicester Cough Questionnaire (LCQ), a self-completed health related quality of life measure of chronic cough.

\section{METHODS}

Patients with chronic cough were recruited from an adult respiratory outpatient clinic. Chronic cough was defined as a cough lasting more than 3 weeks that remained unexplained after assessment by the primary care physician. Patients were investigated and treated using a diagnostic protocol described previously. ${ }^{6}$ All patients with an isolated chronic cough were identified before the outpatient session, during which they were approached by another investigator who had not read the case notes. Patients were asked for their consent to participate in the development of the questionnaire and the protocol was approved by the Leicestershire ethics committee. The LCQ was developed using a multistep method $^{78}$ divided into three phases: phase 1 (item generation); phase 2 (item reduction, allocation of items to domains, and validation of the questionnaire); and phase 3 (repeatability and responsiveness testing of final version of the questionnaire). The item reduction was based on questionnaire responses from 104 patients and concurrent validity was assessed in a separate 56 patients, 27 of whom had a cough sensitivity measurement.

\section{Phase 1: Item generation}

Phase 1 consisted of item generation for a preliminary questionnaire LCQ1 by the following processes: (1) critical review of health related quality of life (HRQOL) literature; (2) review of existing generic and respiratory specific HRQOL questionnaires; (3) multidisciplinary team meeting to generate items for the questionnaire which included respiratory and 
non-specialist doctors, nurse, asthma nurse specialist, physiotherapist, pharmacist and a rehabilitation expert involved in delivering care to patients with chronic cough. A layperson was also present at the meeting; (4) semi-structured interviews with 15 patients with chronic cough to outline areas of concern to them.

Phase 2: Item reduction, allocation of items to domains, and validation of the questionnaire Item reduction

During phase 2, 104 patients with chronic cough were asked to answer each item of the LCQ1 and, in addition, to rate the importance of each item to them on a 5 point scale $(1=$ not important, $5=$ extremely important). Data obtained from the LCQ1 were used to reduce items by the clinical impact factor method $^{9}$ which selects items that are most frequently perceived as important by patients. The mean impact score for each item was calculated as the product of frequency of the item occurring (0-1.00) and mean importance rating of the item. Items were ranked based on their impact scores and an impact score threshold of 1.5 was used to eliminate low ranking items. The following criteria were used to further eliminate items from the questionnaire: (1) high ceiling effect (items with $>60 \%$ of responses falling into the two lowest categories "none of the time" and "hardly any of the time"); (2) the lower impact score items of those with correlation coefficient $>0.8$; (3) items that were similar or ambiguously worded by consensus opinion. The remaining items formed the LCQ2, the final version (appendix 1).

\section{Allocation of items to domains}

Domains for the LCQ were predefined on: (1) World Health Definition of health, ${ }^{10}$ (2) semi-structured interviews held with patients for item selection process, and (3) expert opinion. Based on this, domains were physical, psychological, and social. Each item was allocated to one of the predefined domains using the following criteria: (1) each item's face validity was determined by an expert panel, and (2) each item was correlated with each domain score to ensure that the item was in the most appropriate scale. Internal reliability of each domain was assessed using Cronbach's alpha coefficients which indicate the extent to which the items are interrelated. Internal reliability is generally acceptable for domains with a Cronbach's alpha coefficient of 0.7 or above. ${ }^{8}$

\section{Concurrent validity}

Concurrent validity, which is the assessment of an instrument against other standards that provide an indication of the true value for the measurements, was assessed by correlating scores of LCQ2 with three health outcome measures completed at the same time in 56 patients: (1) cough visual analogue score (VAS) ${ }^{11}$ which has a scale from $0-100 \mathrm{~mm}$ with $100 \mathrm{~mm}$ being the worst imaginable cough, (2) St George's Respiratory Questionnaire (SGRQ) ${ }^{5}$ which includes cough related items, and (3) Short Form 36 item (SF36) health status questionnaire ${ }^{12}$ which is a well tested and validated generic health status measure. The time scale over which the symptoms or events being questioned in the SGRQ or SF36 were adjusted to 2 weeks to be consistent with the LCQ2. Twenty seven patients also had a cough reflex sensitivity measurement using a protocol described elsewhere. ${ }^{13}$ Briefly, capsaicin (a pepper extract) was inhaled in doubling doses via a dosimeter to determine the concentration of capsaicin that causes two and five coughs (C2 and C5). Patients were also asked which of the three questionnaires they found the easiest to complete and relevant to them and the time taken for completion was measured.

\section{Response scale}

A 7 point Likert scale was used throughout the development of the LCQ ranging from $1=$ all of the time to $7=$ none of the time. A higher score indicated better health status. Domains were scored out of 7 (total score from items in domain/number of items in domain). The overall score for the LCQ for each patient was calculated by adding the individual domain scores (appendix 2).

\section{Phase 3: Repeatability and responsiveness testing}

The test-retest procedure measured the stability of scores on the LCQ2 over time in patients who had a stable chronic cough. The LCQ2 and cough VAS was administered in the outpatient clinic. The repeat questionnaire was then mailed to the participants in time for them to complete it 2 weeks after the first questionnaire. Patients were asked to what extent their cough had changed since the first completion of the questionnaire.

Responsiveness of the LCQ2 and cough VAS was tested in the outpatient clinic before and 2 months after a treatment had been commenced. Criteria for starting treatment and individual treatments used were as outlined previously. ${ }^{6}$ Patients were asked if their cough had improved.

\section{Statistical analysis}

SPSS version 10 was used for data analysis. Data are presented as mean (SE) or ranges. Both Pearson correlation coefficients and Spearman rank correlation coefficients were used to determine relationships within and between different outcome measures according to the distribution of the variables. Item to domain correlations were adjusted for over-fitting by removing the score from the item being considered from the total domain score. Agreement between domain and total scores for the first and second completion of the LCQ during repeatability testing were assessed using intraclass correlation coefficients. The $95 \%$ limit of agreement was calculated as 1.96 $\times$ standard deviation (SD) of within subject differences. Effect size for the total LCQ score was determined by the difference in mean total LCQ score before and after the intervention/SD of LCQ total scores before the intervention.

\section{RESULTS}

\section{Phase 1: Item generation}

A review of existing HRQOL questionnaires and literature and multidisciplinary team meeting generated an initial pool of 38 items. A further six items were added after patient interviews. A preliminary questionnaire (LCQ1) comprising 44 items was therefore obtained.

Phase 2: Item reduction, allocation of items to domains, and validation of questionnaire

One hundred and four patients completed the LCQ1. Patients had a mean (range) age of 57 (19-78) years, cough duration of $5(0.2-50)$ years, cough VAS $48(3-96) \mathrm{mm}$, and $39(38 \%)$ were men.

Item reduction

Twenty five of the 44 items were omitted for the reasons given in table 1, which resulted in a questionnaire with 19 items.

\section{Allocation of items to domains}

Eight items were assigned to the physical domain, seven to the psychological domain, and four to the social domain by the expert panel. All items correlated well with their domains (Pearson correlations adjusted for over-fitting 0.4-0.8, all $\mathrm{p}<0.01$ ). Internal consistency was high for all domains as well as the total score (table 2).

\section{Concurrent validity}

Fifty six patients completed the LCQ2 and other outcome measures. Patients had a mean (range) age of 55 (22-85) years, cough duration of $5(0-7)$ years, cough VAS 48 (4-100) $\mathrm{mm}$, and $21(38 \%)$ were men. Correlations between 
Table 1 Reasons for reduction of items in LCQ1

\begin{tabular}{lc}
\hline & $\begin{array}{c}\text { Number of } \\
\text { items (\%) }\end{array}$ \\
\hline $\begin{array}{l}\text { Low ranking items (impact factor } \\
\text { score }<1.5)\end{array}$ & $15(34)$ \\
High ceiling effect & $1(2)$ \\
$\begin{array}{l}\text { Correlation }>80 \% \text { with higher } \\
\text { ranking items }\end{array}$ & $5(11)$ \\
Similar wording & $3(7)$ \\
Ambiguous wording & $1(2)$ \\
Total & $25(57)$ \\
\hline
\end{tabular}

Table 2 Cronbach's alpha coefficients for each domain and total score for Leicester Cough Questionnaire

\begin{tabular}{ll}
\hline Domain & $\begin{array}{l}\text { Cronbach's alpha } \\
\text { coefficient }\end{array}$ \\
\hline Physical & 0.79 \\
Psychological & 0.89 \\
Social & 0.85 \\
Total & 0.92 \\
\hline
\end{tabular}

Table 3 Spearman rank correlations between different health outcome measures and LCQ2

\begin{tabular}{lc}
\hline & $\begin{array}{l}\text { Correlation with LCQ2 } \\
\text { total score }\end{array}$ \\
\hline Cough VAS & -0.72 \\
SGRQ & -0.54 \\
SF36 - total & 0.46 \\
\hline
\end{tabular}

the 19 item LCQ2 and other outcome measures are presented in table 3 . All were highly significant $(p<0.001)$. Correlations did not change significantly when items common to both scales were excluded. Correlations between LCQ and SF36 domains were physical/role limitation due to physical problems $(\rho=0.46)$, psychological/mental health $(\rho=0.59)$, and social/social functioning $(\rho=0.46)$. Pearson's correlations between LCQ total score and cough sensitivity $(\log C 2$ and $\log \mathrm{C} 5)$ were 0.14 and 0.18 , respectively $(\mathrm{p}=0.5$ and 0.4 , respectively, $\mathrm{n}=27$ ). There were no significant correlations between cough sensitivity and LCQ domains or cough VAS. When asked which questionnaire they found easiest to complete, $68 \%$ of patients indicated they preferred the LCQ, $16 \%$ the SF36, $8 \%$ the SGRQ, and $8 \%$ had no preference. The mean time to complete the LCQ was 5 minutes.

\section{Phase 3: Repeatability and responsiveness testing}

Repeatability was tested in 24 patients. Intraclass correlation coefficients for the LCQ domains were: physical 0.93, psychological 0.90, social 0.88, and total score 0.96. A Bland-Altman plot of the LCQ total score is shown in fig 1 . The mean difference (SD) between the first and second LCQ total scores was $0.73(0.94)$. The intraclass correlation coefficient for the cough VAS was 0.84 .

Responsiveness was tested in nine patients who had a specific therapeutic intervention and stated their cough had improved. The cause of cough was gastro-oesophageal reflux $(n=3)$, cough variant asthma $(n=2)$, chronic bronchitis/

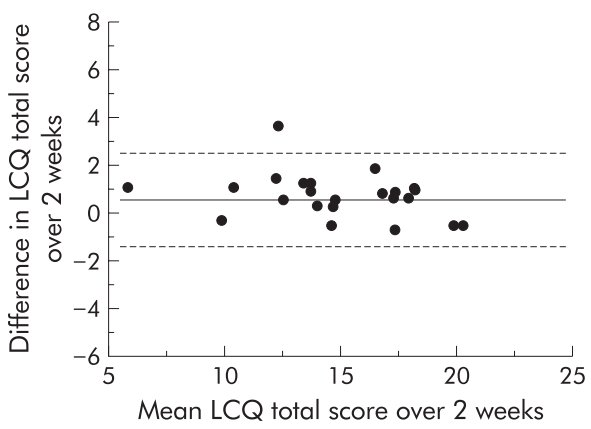

Figure 1 Bland-Altman plot of LCQ total score repeated over 2 weeks in patients whose cough remained unchanged. Solid line represents mean difference and dashed lines represent $95 \%$ limits of agreement.

\begin{tabular}{|c|c|}
\hline \multicolumn{2}{|c|}{$\begin{array}{l}\text { Table } 4 L C Q \text { responsiveness: effect } \\
\text { sizes of } L C Q \text { domains and cough VAS } \\
\text { after treatment }\end{array}$} \\
\hline & Effect sizes $(n=9)$ \\
\hline Physical & 1.00 \\
\hline Psychological & 1.75 \\
\hline Social & 0.84 \\
\hline$L C Q$ total & 1.68 \\
\hline Cough VAS & 3.19 \\
\hline
\end{tabular}

bronchiectasis $(n=2)$, rhinitis $(n=1)$, enlarged tonsils $(n=1)$. Interventions were proton pump inhibitor therapy $(n=3)$, inhaled corticosteroids $(n=3)$, nasal corticosteroid $(n=1)$, tonsillectomy $(n=1)$, and postural drainage $(n=1)$. Changes in cough VAS score were used as a surrogate marker for response to treatment. The mean (SE) change in cough VAS was $-42.3(8.6) \mathrm{mm}$. The effect sizes for change in domain and total LCQ scores are presented in table 4. A $t$ test comparing the change in LCQ total score after treatment was highly significant $(\mathrm{p}=0.007)$.

\section{DISCUSSION}

The LCQ is a valid and reliable health status measure for adults with chronic cough. The final version contains 19 items with a 7 point Likert response scale. It is designed for self-administration and takes less than 5 minutes for completion. $68 \%$ of our patients found our questionnaire easier to complete and more relevant than two other widely used HRQOL measures. This may be because it is a patient derived questionnaire and hence has items, domains, and response scales that are more meaningful to them. The LCQ was highly repeatable and responsive to change, suggesting it might be a particularly useful outcome measure in assessing the response to treatment in the clinic and in trials.

Item reduction was performed using the clinical impact factor method instead of traditional psychometric techniques such as factor analysis. The impact factor chooses items which patients label as a problem and ranks the importance which they associate with them. Items are categorised into domains using clinical sensibility. The factor analysis approach is based largely on the structure of correlations between items, and investigators must also make a number of subjective decisions throughout the process. ${ }^{9}$ Factor analysis does not take into account the perception of clinical relevance of items by the intended population. Instead, item reduction is performed predominantly on complex correlations between items. Many items chosen during questionnaire development will be similar using both techniques, but there are important 
differences. ${ }^{9}$ We share the views of Juniper et al ${ }^{9}$ that all items of functional impairment that are important to patients should be included in a disease specific quality of life health status measure, irrespective of their association with each other.

One limitation of the study was our limited ability to explore the relationship between the LCQ and other objective markers of cough severity. This is due to the lack of well validated outcome measures in chronic cough. However, the LCQ correlated well with the cough VAS and less well with cough sensitivity suggesting that, as in asthma, the relationship between symptoms, quality of life, and physiological impairment is complex. ${ }^{14}$ An absence of correlation between cough symptoms and cough sensitivity has also been noted in patients with cryptogenic fibrosing alveolitis ${ }^{15}$ and chronic obstructive pulmonary disease. ${ }^{16}$ As expected, the LCQ correlated moderately with the SGRQ which has four specific cough items out of many respiratory items and less so with SF36 which focuses on general health alone, providing evidence of concurrent validity.

The LCQ was shown to be highly repeatable over 2 weeks. This was better than the repeatability of other measures of chronic cough such as cough VAS and cough sensitivity. ${ }^{17}$ Our data indicate that a change in total LCQ score of more than 2.56 is likely to be significant since this lies outside the $95 \%$ limit of agreement. We have also shown that the LCQ was responsive to change after treatment although the effect sizes were less than that seen with the cough VAS, suggesting that the latter might be the outcome measure of choice in clinical trials. Effect sizes were comparable to that seen with cough sensitivity measurement in a study where inhaled corticosteroids were given for cough due to eosinophilic bronchitis (effect sizes for C2 and C5: 1.72 and 1.17, respectively). ${ }^{11}$ These characteristics suggest that the LCQ can detect changes in health status as a result of successful treatment.

Since this paper was submitted, French et $a l^{18}$ have described the validation of another cough specific quality of life questionnaire (CQLQ). The LCQ is a briefer questionnaire with fewer domains than the CQLQ which comprises 28 items and six domains. The latter questionnaire also differs from ours in that it used factor analysis for allocation of items to domains, subjective criteria for item reduction, and provided more limited information on concurrent validity against other measures. Further work is necessary to compare the LCQ and CQLQ in the assessment of chronic cough in European and North American populations.

This study suggests that the LCQ will be suitable for a number of applications. Firstly, it would be useful in describing longitudinal changes that take place in patients with chronic cough. It can be used to identify aspects of health affected by cough and how these change over time. Finally, it can be used in clinical trials evaluating new treatments for cough and their effect on health related quality of life. In summary, the LCQ is a brief, easy to administer, and well validated chronic cough HRQOL questionnaire. It represents an advance in the management of chronic cough where there is a lack of objective measures to guide the clinician and scientist.

\section{APPENDIX 2: Scoring of LCQ}

(1) Domains (questions):

(a) Physical: 1,2,3,9,10,11,14,15

(b) Psychological: 4,5,6,12,13,16,17

(c) Social: 7,8,18,19

(2) Domain scores: total score from items in domain/number of items in domain (range $1-7$ ).

(3) Total scores: addition of domain scores (range 3-21).

\section{ACKNOWLEDGEMENTS}

The authors would like to thank Paula Billington, Wendy Chawner and the outpatient clinic staff for their help in administering the questionnaire.

\section{Authors' affiliations}

S S Birring, B Prudon, S J Singh, M D L Morgan, I D Pavord, Institute for Lung Health, Department of Respiratory Medicine, Glenfield Hospital, Leicester, UK

A J Carr, Musculoskeletal Epidemiology, Department of Academic Rheumatology, University of Nottingham, Nottingham City Hospital, Nottingham, UK

Funding: British Lung Foundation and University Hospitals of Leicester NHS Trust. Surinder Birring is a British Lung Foundation Clinical Research Fellow.

Conflicts of interest: none declared.

Reprints of LCQ: the Leicester Cough Questionnaire $\odot 2001$ is protected by copyright. Reprints of the $L C Q$ are available from the corresponding author.

\section{REFERENCES}

1 Fuller RW, Jackson DM. Physiology and treatment of cough. Thorax 1990;45:425-30

2 Irwin RS, Madison JM. The diagnosis and treatment of cough. N Engl J Med 2000;343:1715-21.

3 French CL, Irwin RS, Curley FJ, et al. Impact of chronic cough on quality of life. Arch Intern Med 1998;158:1657-61.

4 Juniper EF, Guyatt GH, Ferrie PJ, et al. Measuring quality of life in asthma. Am Rev Respir Dis 1993;147:832-8.

5 Jones PW, Quirk FH, Baveystock CM, et al. A self-completed measure for chronic airflow limitation: the St George's Respiratory Questionnaire. Am Rev Respir Dis 1992;145:1321-7.

6 Brightling CE, Ward R, Goh KL, et al. Eosinophilic bronchitis is an important cause of cough. Am J Respir Crit Care 1999;160:406-10.

7 Guyatt GH, Bombardier C, Tugwell PX. Measuring disease-specific quality of life in clinical trials. Can Med Assoc J 1986;134:889-95.

8 Fayers PM, Machin D. Quality of life: assessment, analysis and interpretation. Wiley Publishers, 2000: 43-71.

9 Juniper EF, Guyatt GH, Streiner DL, et al. Clinical impact versus factor analysis for quality of life questionnaire construction. J Clin Epidemiol 1997;50:233-8.

10 World Health Organisation. Constitution of the World Health Organisation. Geneva: WHO, 1947.

11 Brightling CE, Ward R, Wardlaw AN, et al. Airway inflammation, airway responsiveness and cough before and after inhaled budesonide in patients with eosinophilic bronchitis. Eur Respir J 2000;15:682-6.

12 Brazier JE, Harper R, Jones NMB, et al. Validating the SF36 health survey questionnaire: new outcome measure for primary care. $B M$ 1992;305:160-4.

13 Choudry NB, Fuller RW. Sensitivity of the cough reflex in patients with chronic cough. Eur Respir J 1992;5:296-300.

14 Moy ML, Israel E, Weiss ST, et al. Clinical predictors of health-related quality of life depend on asthma severity. Am J Respir Crit Care 2001;163:924-9.

15 Doherty MJ, Mister R, Pearson MG, et al. Capsaicin induced cough in cryptogenic fibrosing alveolitis. Thorax 2000;55:1028-32.

16 Doherty MJ, Mister R, Pearson MG, et al. Capsaicin responsiveness and cough in asthma and chronic obstructive pulmonary disease. Thorax 2000;55:643-9

17 Chang AB, Phelan PD, Roberts RGD, et al. Capsaicin cough receptor sensitivity test in children. Eur Respir J 1996;9:2220-3.

18 French CT, Irwin RS, Fletcher KE, et al. Evaluation of a cough-specific quality-of-life questionnaire. Chest 2002;121:1123-31. 
This questionnaire is designed to assess the impact of cough on various aspects of your life. Read each question carefully and answer by CIRCLING the response that best applies to you. Please answer ALL questions, as honestly as you can.

1. In the last 2 weeks, have you had chest or stomach pains as a result of your cough?

$\begin{array}{lllllll}1 & 2 & 3 & 4 & 6 & 7 \\ \text { All of the time } & \text { Most of the time } & \text { A good bit of the time } & \text { Some of the time } & \text { A little of the time } & \text { Hardly any of the time } & \text { None of the time }\end{array}$

2. In the last 2 weeks, have you been bothered by sputum (phlegm) production when you cough?

\begin{tabular}{|c|c|c|c|c|c|}
\hline & 2 & 3 & 4 & & \\
\hline Every time & Most times & Several times & Some times & Occasionally & Rarely \\
\hline
\end{tabular}

3. In the last 2 weeks, have you been tired because of your cough?

$\begin{array}{lllllll}1 & 2 & 3 & 4 & 6 & & 7 \\ \text { All of the time } & \text { Most of the time } & \text { A good bit of the time } & \text { Some of the time } & \text { A little of the time } & \text { Hardly any of the time } & \text { None of the time }\end{array}$

4. In the last 2 weeks, have you felt in control of your cough?

$\begin{array}{llllllll}1 & 2 & 3 & 5 & 6 & 7 \\ \text { None of the time } & \text { Hardly any of the time } & \text { A little of the time } & \text { Some of the time } & \text { A good bit of the time } & \text { Most of the time } & \text { All of the time }\end{array}$

5. How often during the last 2 weeks have you felt embarrassed by your coughing?

$\begin{array}{lllllll}1 & 2 & 3 & 4 & 6 & & 7 \\ \text { All of the time } & \text { Most of the time } & \text { A good bit of the time } & \text { Some of the time } & \text { A little of the time } & \text { Hardly any of the time } & \text { None of the time }\end{array}$

6. In the last 2 weeks, my cough has made me feel anxious

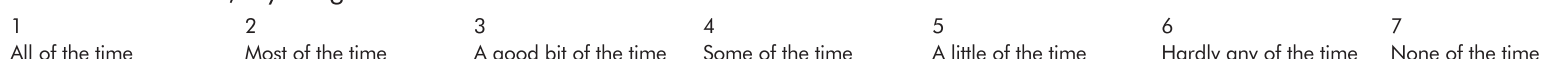

7. In the last 2 weeks, my cough has interfered with my job, or other daily tasks

$\begin{array}{llllll}1 & 2 & 3 & 4 & 6 & 7 \\ \text { All of the time } & \text { Most of the time } & \text { A good bit of the time } & \text { Some of the time } & \text { A little of the time } & \text { Hardly any of the time }\end{array}$

8. In the last 2 weeks, I felt that my cough interfered with the overall enjoyment of my life

$\begin{array}{llllll}1 & 2 & 3 & 4 & 6 & 7 \\ \text { All of the time } & \text { Most of the time } & \text { A good bit of the time } & \text { Some of the time } & \text { A little of the time } & \text { Hardly any of the time }\end{array}$

9. In the last 2 weeks, exposure to paints or fumes has made me cough

$\begin{array}{lllllll}1 & 2 & 3 & 5 & 6 & 7 \\ \text { All of the time } & \text { Most of the time } & \text { A good bit of the time } & \text { Some of the time } & \text { A little of the time } & \text { Hardly any of the time } & \text { None of the time }\end{array}$

10. In the last 2 weeks, has your cough disturbed your sleep?

$\begin{array}{llllll}1 & 2 & 3 & 5 & 6 & 7 \\ \text { All of the time } & \text { Most of the time } & \text { A good bit of the time } & \text { Some of the time } & \text { A little of the time } & \text { Hardly any of the time }\end{array}$

11. In the last 2 weeks, how many times a day have you had coughing bouts?
1 All of the time
2 Most times durin
3 Several times during 4 Some times during (continuously)
$\begin{array}{lll}2 & \text { Most times during } 3 \text { Several times during } 4 \text { Some tim } \\ \text { the day } & \text { the day }\end{array}$
5 Occasionally through
the day Rarely
7

12. In the last 2 weeks, my cough has made me feel frustrated

\begin{tabular}{|c|c|c|c|c|c|c|}
\hline & 2 & 3 & 4 & 5 & 6 & 7 \\
\hline All of the time & Most of the time & A good bit of the time & Some of the time & A little of the time & Hardly any of the time & None of the time \\
\hline & & & & & & \\
\hline 1 & 2 & 3 & 4 & 5 & 6 & 7 \\
\hline
\end{tabular}

14. In the last 2 weeks, have you suffered from a hoarse voice as a result of your cough?

\begin{tabular}{|c|c|c|c|c|c|c|}
\hline $\begin{array}{l}1 \\
\text { All of the time }\end{array}$ & $\begin{array}{l}2 \\
\text { Most of the time }\end{array}$ & 3 & $\begin{array}{l}4 \\
\text { Some of the time }\end{array}$ & $\begin{array}{l}5 \\
\text { A little of the time }\end{array}$ & 6 Hardlvany ofthe time & $\begin{array}{l}7 \\
\text { None of the time }\end{array}$ \\
\hline \multicolumn{7}{|c|}{ 5. In the last 2 weeks, have you had a lot of energy? } \\
\hline 1 & 2 & 3 & 4 & 5 & 6 & 7 \\
\hline None of the time & Hardly any of the time & A little of the time & Some of the time & A good bit of the time & Most of the time & All of the time \\
\hline \multicolumn{7}{|c|}{ 6. In the last 2 weeks, have you worried that your cough may indicate serious illness? } \\
\hline 1 & 2 & 3 & 4 & 5 & 6 & 7 \\
\hline All of the time & Most of the time & A good bit of the time & Some of the time & A little of the time & Hardly any of the time & None of the time \\
\hline
\end{tabular}

17. In the last 2 weeks, have you been concerned that other people think something is wrong with you, because of your cough?

\begin{tabular}{|c|c|c|c|c|c|c|}
\hline All of the time & $\begin{array}{l}2 \\
\text { Most of the time }\end{array}$ & $\begin{array}{l}3 \\
\text { A good bit of the time }\end{array}$ & $\begin{array}{l}4 \\
\text { Some of the time }\end{array}$ & 5 & 6 & 7 \\
\hline
\end{tabular}

18. In the last 2 weeks, my cough has interrupted conversation or telephone calls

$\begin{array}{llllll}1 & 2 & 3 & 5 & 6 & 7 \\ \text { Every time } & \text { Most times } & \text { A good bit of the time } & \text { Some of the time } & \text { A little of the time } & \text { Hardly any of the time }\end{array}$

19. In the last 2 weeks, I feel that my cough has annoyed my partner, family or friends
Every time I cough
2 Most times when
3 Several
I cough
4 Some tim
I cough
5 Occasionally when
Rarely
7

Thank you for completing this questionnaire. 